\title{
Die intraligamentäre Anästhesie - Primäre Methode der dentalen Lokalanästhesie
}

Seit Jahrzehnten wird die intraligamentäre Anästhesie als Lokalanästhesie-Methode allenfalls in einer Minderheit der Praxen eingesetzt. Wenn man sie sich nicht auf einem Fortbildungskurs angeeignet hat, dann hat man sie sich selbst beigebracht. Meist wird sie nur angewandt, wenn die Infiltrations- oder die Leitungsanästhesie nicht zum Erfolg geführt haben. Denn: Systematisch gelehrt wird sie an den Universitäten nicht. Das hat mit einer Kollision von „Meinungen“ und Fakten zu tun. Man meint, genug Gründe zu haben, auf eine Lehre zu verzichten, ohne aber die Fakten zu kennen. Lehr-Meinung ist: diese Methode nicht zu lehren. Wie sinnfrei ist es, über diese - seit mehr als 100 Jahren bekannte - Möglichkeit der Einzelzahnanästhesie mit bloßen Meinungen statt durch Studien belegte Fakten zu argumentieren?

Abhilfe schafft das Fachbuch „Die intraligamentäre Anästhesie - Primäre Methode der dentalen Lokalanästhesie“von Benz, Prothmann und Taubenheim. Darin werden weitgehend alle Aspekte der ILA zusammengefasst. Als Alternative zur Infiltrations- und Leitungsanästhesie werden die Grenzen und Komplikationen auch dieser Lokalanästhesie-Methoden beschrieben und damit die Möglichkeit eröffnet, die ILA als primäre Methode der zahnärztlichen Lokalanästhesie anzuwenden. Die Autoren stellen dar, dass es keine haltbaren Gründe mehr gibt, warum Leitungs- und Infiltrationsanästhesie allgemein gelehrt und praktiziert werden, den Patienten die intraligamentäre Anästhesie aber noch immer vorenthalten wird.

\section{Mehr als 200 wissenschaftlich relevante Quellen}

Den aktuellen Stand von Wissenschaft und Technik zur ILA unter Einbeziehung der veröffentlichten Studienergebnisse aus Klinik und Praxis von 1920 bis 2014 haben Christoph Benz, Poliklinik für Zahnerhaltung und Parodontologie der Universität München, Marc Prothmann, niedergelassen in eigener Praxis in Berlin und Lothar Taubenheim, Medizinjournalist VMWJ und seit Jahren mit der systematischen Aufarbeitung der Methode der ILA befasst, umfassend analysiert. Sie haben das vorhandene Wissen zusammengetragen, die vorliegenden Studien betrachtet, sortiert und für Studierende und Praktiker zugänglich gemacht. So werden im Literaturverzeichnis mehr als 200 wissenschaftlich relevante Quellen angegeben. Mehr als 70 Fachbeiträge zur ILA wurden in den vergangenen zehn Jahren in renommierten wissenschaftlichen Fachzeitschriften international publiziert. Übrigens: Erstmals war die ILA umfassenderes Thema beim Zahnärztetag 2015. Insbesondere die aktuelle Rechtsprechung zur Aufklärungspflicht über Risiken und Alternativen der Leitungsanästhesie rückt die intraligamentäre Anästhesie aus ihrem Schattendasein heraus - als Alternative zu den täglich angewandten Methoden der Schmerzausschaltung.

Der haftungsrechtlich wichtige Hinweis auf diese Aufklärungspflicht sollte keinesfalls auf die leichte Schulter genommen werden. Ein wesentlicher Aspekt wird von den Autoren sehr deutlich dargestellt: Der Erfolg der ILA hängt maßgeblich von den verwendeten - dem Stand der Medizintechnik entsprechenden - Instrumentarien, der Applikation bewährter Anästhetika (mit Adrenalin) und der sicheren Beherrschung dieser bisher nicht systematisch gelehrten Lokalanästhesie-Methode ab. Wenn diese Kriterien erfüllt sind, treten praktisch kaum iatrogene Nebenwirkungen, wie Elongationsgefühl, Druckschmerz oder Vorkontakt nach Abklingen der Analgesie, oder auch reversible Drucknekrosen auf. Das Risiko, bei der Insertion der Kanüle ins Ligamentum circulare ein Gefäß oder einen Nervstrang zu treffen, ist nicht gegeben, weil es im Desmodontalspalt solche nicht gibt.

\section{Basislektüre für Ausbildung und Praxis}

In dem gut gegliederten Fachbuch des Deutschen Zahnärzte Verlags werden in modernem Layout in sehr verständlicher Form alle in Betracht kommenden Indikationen, die bekannten ungewünschten Effekte und allem voran auch die praktische Anwendung der intraligamentären Anästhesie detailliert beschrieben. Die verschiedenen Injektionssysteme, für die es belastbare Studien gibt, werden vorgestellt, darunter auch ein High-Tech-System. Der Band erfüllt alle Kriterien einer Basislektüre für die zahnärztliche Ausbildung, aber auch für alle praktizierenden Zahnärzte. Die Autoren kommen zu der Aussage, dass es keinen vertretbaren Grund mehr gibt, die intraligamentäre Anästhesie - neben Extraktionen und Osteotomien - nicht in der täglichen Praxis als primäre Methode der Lokalanästhesie auch für die Schmerzausschaltung vor zahnerhaltenden, endodontischen und parodontologischen (geschlossenes Vorgehen) Therapien einzusetzen. Dieser Meinung kann sich der Rezensent aus eigener Erfahrung guten Gewissens anschließen.

Carlheinz Swaczyna

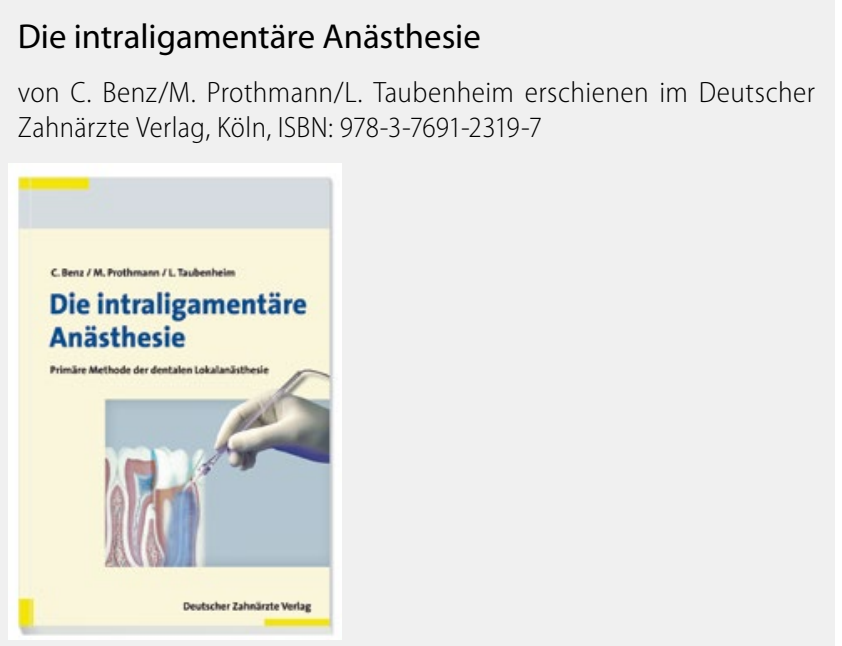

